
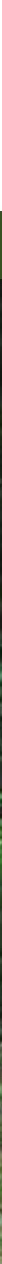

\title{
Votre proposition traduit-elle une attention suffisante portée à la problématique du genre ?
}

Nous demandons à présent aux chercheurs et chercheuses du CIFOR d'envisager sérieusement si le genre est à prendre en considération dans leurs travaux, et si oui de quelle façon. La prise en compte du genre dans la recherche est un élément fondamental de la réalisation de travaux scientifiques de qualité. C'est un outil dont on dispose pour faire en sorte que la recherche forestière conduise à des progrès socialement équitables dans le bien-être humain et la conservation de l'environnement. La prise en compte du genre vous permettra de rédiger des propositions plus concurrentielles et de générer des résultats de recherche plus pertinents et durables. Ce guide synthétique fournit des questions essentielles à vous poser afin que la proposition que vous adresserez au CIFOR témoigne d'une prise en considération adéquate de la problématique du genre.

La recherche sensible aux questions de genre étudie les priorités et les besoins différents des hommes et des femmes. Elle analyse l'influence des rapports de genre sur la capacité des hommes et des femmes de gérer et d'utiliser les forêts et les produits forestiers, ainsi que les effets différenciés des politiques menées sur les hommes et les femmes. La recherche vise à cerner les causes fondamentales des inégalités de genre. Elle englobe la collecte de données sexuées et l'analyse des inégalités de genre dans le but d'étudier leurs incidences sur différents groupes sociaux. La recherche axée sur le genre est le plus souvent menée en collaboration avec les communautés et d'autres acteurs qui participent à la définition de son champ d'action et de ses activités. Les recommandations découlant de cette recherche détermineront les améliorations à apporter aux politiques et pratiques forestières qui seront les plus intéressantes pour toutes les parties, dans le but de corriger l'inégale répartition des ressources et le déséquilibre de pouvoir entre les hommes et les femmes.

\section{Buts et objectifs}

Les buts et objectifs définis lors de la conception de vos travaux doivent mettre en évidence en quoi leur accomplissement concourt à favoriser l'équité et à réduire la pauvreté, en harmonie avec la mission du CIFOR et les Résultats sur le plan systémique (System-Level Outcomes) du CGIAR.

Il convient au minimum que les buts, objectifs et résultats se réfèrent expressément aux hommes et aux femmes et non simplement aux «agriculteurs» ou aux «villageois». Mais vous pourriez vous efforcer de formuler les buts, les objectifs et les questions de recherche de façon à démontrer que le genre est une variable analytique et explicative essentielle au travers de laquelle il est possible d'étudier d'autres variables sociales. Tous les projets ne peuvent comporter une perspective de genre. Néanmoins, il est important de montrer que vous avez réfléchi à la manière dont vos travaux peuvent concourir à favoriser l'équité ou utiliser des outils d'analyse axée sur le genre (Encadré 1).

- Avez-vous montré comment vous allez prendre en compte ou privilégier la problématique du genre dans vos travaux? La façon dont vous formulerez les buts, 


\section{Encadré 2. Où trouver des questions de recherche liées au genre?}

Consultez les ressources suivantes:

- CIFOR. 2013. Gender in the CGIAR Research Programme on Forests, Trees and Agroforestry: A Strategy for research and action. CIFOR, Bogor, Indonésie.

- Manfre, C. et Rubin, D. 2012. Intégrer le genre en recherche forestière : guide pour les scientifiques et les administrateurs de programme du CIFOR. CIFOR, Bogor, Indonésie.

- CIFOR. 2011. CGIAR Research Programme 6: Forests, Trees and Agroforestry: Livelihoods, Landscapes and Governance. Proposal. CIFOR, Bogor, Indonésie.

le genre portant sur les rapports hommes-femmes exige des données significatives et valables sur les femmes et les hommes, et pas seulement sur l'un ou l'autre de ces groupes.

- $\quad$ Avez-vous réfléchi aux méthodes les plus adaptées à l'échelle de vos travaux? Si vous effectuez une comparaison entre pays, les enquêtes pourraient être plus appropriées, tandis que les méthodes participatives pourraient être surtout utiles pour étudier l'évolution de l'accès ou des droits liés à la forêt dans une région donnée. Les approches participatives et collaboratives sont en général mieux adaptées aux activités de coopération étroite avec les communautés locales ou d'autonomisation de celles-ci.

- Avez-vous réfléchi aux méthodes les plus adaptées au public ciblé par vos recherches?

- Avez-vous envisagé la nécessité de recourir à des interviewers ou des enquêteurs masculins et féminins? En fonction du contexte culturel de vos travaux, les hommes doivent interviewer les hommes et/ou les femmes doivent interviewer les femmes.

- Avez-vous réfléchi à la nécessité de prévoir des activités participatives telles qu'interviews et groupes de discussion en groupes mixtes et/ou unisexes?

\section{Encadré 3. Faites attention à la composition hommes-femmes de votre équipe.}

Favoriser la parité dans les métiers scientifiques et de la recherche est un objectif important pour le CIFOR et le CGIAR. De nombreux donateurs, tels I'UE, accordent une attention sans cesse croissante à l'intégration du genre dans les contenus scientifiques, mais aussi à la manière dont les établissements de recherche impulsent l'égalité des chances dans leur politique et leur pratique. L'inclusion de chercheuses dans votre équipe ne garantira pas que vos travaux ou résultats de recherche prennent mieux en compte les spécificités de genre. Mais promouvoir l'égalité des chances de participation des hommes et des femmes à la recherche permet de franchir une première étape dans l'instauration d'une culture sur les lieux de travail qui permette tant aux acteurs qu'aux actrices de s'épanouir.

\subsection{Partenariats et alliances}

- Avez-vous inclus dans vos partenaires de recherche des associations de femmes ou d'autres organisations de promotion de l'égalité entre les sexes ou de défense des droits des femmes? Vous pouvez établir des partenariats et des alliances avec les comités nationaux ou régionaux et les ministères de l'identité de genre ou de la femme, ou les associations de femmes ou de femmes entrepreneurs.

- $\quad$ Avez-vous envisagé de créer des partenariats avec des associations de femmes ou d'autres organisations de promotion de l'égalité entre les sexes ou de défense des droits des femmes dans le cadre d'activités de sensibilisation ou d'approche des groupes marginalisés?

\subsection{Communication des connaissances pour la définition de politiques et l'action}

- Avez-vous expliqué clairement comment vous allez incorporer les statistiques, tableaux, figures, descriptions et analyses de données sexuées?

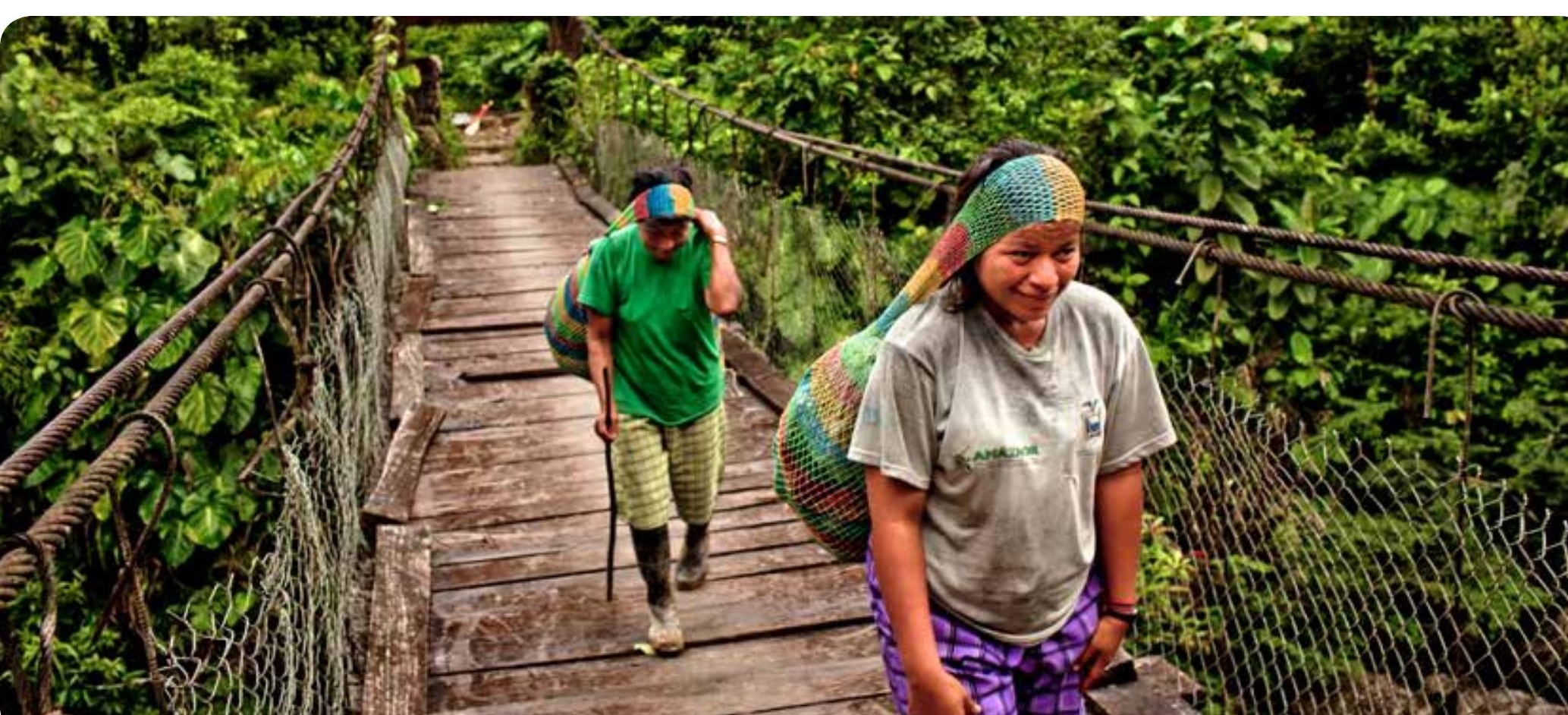


- $\quad$ Avez-vous envisagé de produire un livrable, une publication ou un événement précis pour présenter vos résultats selon le genre?

- $\quad$ Avez-vous prévu les modalités de diffusion des résultats de votre recherche auprès de ses bénéficiaires, dont les femmes? Quand vous rendez compte de vos travaux aux communautés, assurez-vous que les femmes peuvent avoir accès aux résultats et les comprendre.

- $\quad$ Avez-vous réfléchi à la façon dont vous allez communiquer vos connaissances aux associations ou comités de femmes ou aux autres organisations de promotion de l'égalité entre les sexes ou de défense des droits des femmes?

\section{Renforcement des capacités}

- $\quad$ Avez-vous réfléchi à la nécessité de former les membres de votre équipe de recherche afin qu'ils aient une conception commune de ce qu'est le genre?

- Avez-vous réfléchi à la nécessité de former les enquêteurs et les enquêtrices en analyse selon le genre?

- Avez-vous réfléchi à la nécessité d'organiser un atelier sur les outils et les méthodes d'analyse selon le genre?

- $\quad$ Avez-vous trouvé des experts ou des organisations intervenant sur la problématique du genre qui pourraient accompagner votre équipe tout au long du processus de recherche?

\section{Apprentissage adaptatif}

- Vous êtes-vous assurés que vos indicateurs sur le plan humain sont sexués? Cela veut dire que la décomposition des données ne doit pas s'arrêter au niveau du ménage. Votre recherche doit décomposer selon le sexe tout indicateur de suivi de nombres ou de pourcentages relatifs à des personnes.

- $\quad$ Avez-vous inclus des indicateurs sexués au niveau des résultats afin de mesurer l'évolution des connaissances, des capacités ou des comportements des populations ciblées?

- Avez-vous envisagé d'utiliser les indicateurs de mesure des principaux impacts liés au genre mentionnés dans le Programme de recherche sur les forêts, les arbres et I'agroforesterie du CGIAR ? Ceux-ci comprennent :

- La progression de l'égalité entre les sexes dans la prise des décisions relatives aux forêts, aux arbres et aux ressources agroforestières et dans le contrôle de ceux-ci, et l'amélioration des revenus et des avantages revenant aux femmes grâce à des chaînes de valeur adaptées ;

- L'augmentation du nombre de femmes ayant acquis l'autonomie suffisante pour planter et gérer les forêts, les arbres et les ressources agroforestières dans les pays du programme ;

- La diminution des inégalités de revenus entre les hommes et les femmes en ce qui concerne les revenus tirés des biens et services liés aux forêts, aux arbres et à l'agroforesterie ;

- Les meilleures conditions sanitaires et la diminution de l'incidence des maladies banales due aux carences nutritionnelles parmi les femmes et leurs familles;

- L'augmentation du nombre de femmes parmi les responsables élus/nommés dans les comités de gestion des forêts ;

- Le contrôle accru exercé par les femmes (c.-à-d. le renforcement de leurs droits) sur les forêts, les arbres et les ressources agroforestières à l'échelle du ménage et de la communauté.

- Avez-vous inclus des mesures d'évaluation de l'efficacité du processus de prise en compte du genre ? Le CIFOR s'intéresse non seulement à la détection des produits et des résultats différenciés selon le genre, mais aussi à la mise en évidence de la plus ou moins grande efficacité de la prise en compte du genre dans le processus de recherche. Vous pourriez envisager d'inclure des indicateurs de mesure des progrès accomplis dans le développement des capacités, notamment le nombre de formations dispensées ou l'amélioration de la connaissance des questions de genre.

\section{Budget}

Lors de l'établissement de votre budget, soyez explicites à propos des ressources financières nécessaires aux activités relatives au genre. Plus vous serez précis dans la définition des activités et des produits, plus vous pourrez déterminer les ressources financières nécessaires à leur exécution.

- $\quad$ Avez-vous mis de côté suffisamment de fonds pour réaliser les activités relatives au genre que vous avez prévues, que ce soit la collecte de données ou la communication et la diffusion de connaissances? Vous pourriez avoir besoin d'inclure les dépenses associées à la conduite d'enquêtes à l'intérieur des ménages, à l'organisation de groupes de discussion distincts pour les hommes et les femmes ou à l'engagement d'un spécialiste du genre dans votre équipe.

- Avez-vous intégré les dépenses liées au renforcement des capacités?

- Vous pourriez avoir besoin de prévoir des crédits pour la formation à la problématique du genre, ceci afin de renforcer les capacités de votre équipe de recherche, de vos enquêteurs et enquêtrices ou de vos partenaires.

- Avez-vous prévu des fonds suffisants dans votre budget pour le suivi et l'évaluation des impacts de vos travaux sur les bénéficiaires féminins et masculins?
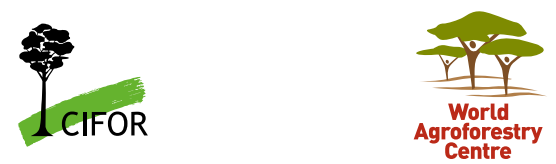

\section{cifor.org/forests-trees-agroforestry}

\section{․ㅏAT} cirad
Cette recherche a été menée par le CIFOR dans le cadre du Programme de recherche du CGIAR sur les forêts, les arbres et l'agroforesterie (CRP-FTA). Ce programme collaboratif vise à améliorer la gestion et l'utilisation des forêts, de l'agroforesterie et des ressources génétiques des arbres à l'échelle du paysage, des forêts aux exploitations agricoles. Le CIFOR dirige le CRP-FTA en partenariat avec Bioversity International, le CIRAD, le Centre international d'agriculture tropicale et le Centre mondial de I'Agroforestrie. 